\title{
Quantification of Physiological Disparities and Task Performance in Stress and Control Conditions
}

\author{
Ahmad Rauf Subhani, Likun Xia, Aamir Saeed Malik and Zahiruddin Othman
}

\begin{abstract}
In mental stress studies, cerebral activation and autonomic nervous system are important distinctly. This study aims to analyze disparities associated with scalp potential, which may have impact on autonomic activation of heart during mental stress. Ten healthy subjects participated in this study that performed arithmetic tasks in stress and control environment. Task difficulty was calculated from their correct responses. During the experiment, electroencephalogram (EEG) and electrocardiogram (ECG) signals were recorded concurrently. Sympathetic innervation of heart was estimated from heart rate (HR), which is extracted from the ECG. The value of theta $\mathrm{Fz} / \mathrm{alpha} \mathrm{Pz}$ was measured from EEG scalp potential. The results show a significant surge in the value of theta Fz/alpha $\mathrm{Pz}$ in stress as compared to baseline $(\mathbf{p}<\mathbf{0 . 0 1 3})$ and control $(p<0.042)$. The results also present tachycardia while in stress as compared to baseline $(p<0.05)$. Task difficulty in stress is also considerably higher than control environment $(\mathbf{p}<0.003)$.
\end{abstract}

\section{INTRODUCTION}

Stress has a common recognition of a state when an individual is expected too much under sheer pressure that he/she is hardly able to deal with demands. These demands can be social or personal. It is known that psychological and social stress exist in daily life, which has affected people's emotional behavior, job performance, mental and physical health and ultimately quality of life. Latest neuroscience reveals that human brain is the main target of mental stress [1] because perception of human brain determines a situation threatening and stressful. Cognitive triggering such as mental stress influences autonomic innervation of the heart. This cardiac innervation can be complicated and fatal in extreme case. The brain is connected with the heart through autonomic nervous system (ANS). ANS indicates expressions of mental activation during stress in initial stages. ANS has two branches: sympathetic nervous system (SNS) and parasympathetic nervous system (PSNS). Heart has excitatory and inhibitory behavior under sympathetic and parasympathetic innervations, respectively [2]. Sympathetic innervation increases myocardial depolarization which can cause tachycardia. However, parasympathetic innervation reduces myocardial depolarization which

Ahmad Rauf Subhani, *Likun Xia and Aamir Saeed Malik are with the Centre for Intelligent Signal and Imaging Research, Department of Electrical and Electronics Engineering, Universiti Teknologi PETRONAS, Tronoh, Perak 31750 Malaysia (phone: +605-368-7828; fax: +605-3657443; e-mail: likun_xia@petronas.com.my).

Zahiruddin Othman is with the Department of Psychiatry, School of Medical Sciences, Univesiti Sains Malaysia, Kubang Kerian, Kelantan 16150 Malaysia. ultimately causes bradycardia and reduces the heart rate (HR).

Electroencephalography (EEG) measures scalp potential noninvasively. EEG signals are rich in providing information about the brain dynamics. Frequency spectrum of EEG signals is divided in different frequency bands: delta $(\delta)(1-4 \mathrm{~Hz})$, theta $(\theta)(4-8 \mathrm{~Hz})$, alpha $(\alpha)(8-12 \mathrm{~Hz})$, beta $(\beta)$ $(12-30 \mathrm{~Hz})$ and gamma $(\gamma)(30-40 \mathrm{~Hz})$. These frequency bands represent many behaviors of brain, e.g., high demand and task difficulty activates theta. Similarly calmness of the brain is narrated by the activation in alpha. Furthermore, scalp locations beneath the EEG electrodes also illustrate useful information because every brain location is responsible of exclusive activities. For example, frontal midline $(\mathrm{Fz})$ performs function of motor planning and parietal midline $(\mathrm{Pz})$ is involved in perceptions [3]. An increasing task load is related to enhanced theta activity in $\mathrm{Fz}$ and reduced alpha activity in $\mathrm{Pz}$ [4].

Electrocardiography (ECG) is noninvasive in use and reflects variations in HR effectively. It keeps track of cardiac adaptation in internal and external variations. Previous studies have reported changes in cardiac activities such as $\mathrm{HR}$, heart rate variability (HRV) and blood pressure in situations such as mental workload, task performance, and exercises [5-7]. Similar situation is also stated to trigger brain activation [4, 8-10]. In [5], HR was measured to discriminate high mental stress from mild stress and no stress during car driving. The results showed a discrete difference between stress and non-stress conditions. HR was also analyzed in [6] to measure cognitive load and mental stress. Moreover, study [7] analyzed HRV to evaluate mental stress among positive responders, negative responders and non-responders.

Scalp potential, HR and HRV were measured in [8] to show correlation between laterality ratio at parietal region (P3 vs. P4) and HR changes. In EEG studies, increased demands in terms of working memory along with high workload, can increase frontal theta power and decrease parietal alpha power [11-13]. Fronto-parietal association was found as a measure of workload index [4, 9], which showed significant relationship with increased task difficulty. EEG and HRV were analyzed in $[12,13]$ during video game playing. Results showed an increment in SNS activity during game playing along with increase task difficulty as represented by EEG signals. 
During our previous work [12], the synchronization of EEG scalp potential and ANS control on heart has been discussed. The current study extends the concept towards the measurement of mental stress. It investigates how information from EEG scalp potential, in terms of theta Fz/alpha $\mathrm{Pz}(\operatorname{Fz}(\theta) / \mathrm{Pz}(\alpha))$, is related to the changes in $\mathrm{HR}$ under mental stress. For this purpose, a paradigm based on Montreal Imaging Stress Task (MIST) is designed to elicit psychosocial stress. MIST was originally designed to induce and evaluate mild psychological stress in terms of physiology and brain activation in functional magnetic resonance imaging (fMRI) environment [1]. In this paper, we aim at analyzing physiological activation using EEG and ECG. This study also uses subject's performance of solving trials in experiment to measure task difficulty.

\section{Methodology}

\section{A. Experiment and Subjects}

The experimental process consisted of six sequential phases, as shown in fig 1 . First phase was drill where a subject performs training of solving sample questions. Next phase was sensors placement and impedance checking. After sensors placement, the third phase was habituation for five minutes; time provided for the subject to get used to of the environment. Recording of physiological signals was not started. It starts from the fourth phase, i.e., rest 1 . The rest phase began with looking at the circle in the centre of the screen for five minutes and was considered as a baseline. Fifth phase was the task phase for twenty minutes in which the subject needed to solve experimental tasks. The last phase was rest 2 . This was the recovery phase which was similar to rest 1 in which the subject needed to stare at a circle in the centre of the screen.

The complete experiment had two sessions: stress and control. In both the sessions, the same experimental process was repeated with the difference lying in the task phase. The core of both sessions was same, that was, to solve mental arithmetic. The difference was that in stress condition time was limited to solve the arithmetic task along with stressful feedbacks and statements. However, there was no such time limitation and feedback in control condition. The minimum duration between two sessions was seven days in order to minimize learning effect. In order to eliminate the expected effect of these sessions on results, half of the subjects appeared in the stress session followed by the control session and the other half of the subjects appeared in control session followed by stress condition. The task phase in stress and control sessions was divided into four levels of increasing difficulty as shown in fig. 2. Duration of each level was five minutes. In every level, multiple trials of same difficulty were repeated. In stress condition, duration to solve a trial was fixed. After the trial, a feedback appeared displaying the words "correct", "incorrect" or "no response" based on correct/incorrect response or no response of the arithmetic question. In stress condition, the feedback display also showed the average performance of a particular level as well as the response time in order to induce more pressure on the subject. However, there was no such feedback display in control condition except of showing correct or incorrect display. Moreover, in stress condition, after certain number of trials in every level, a stressful interrupt popped up showing some stimulating statements such as "Don't guess answers", "Your performance is below average" etc.

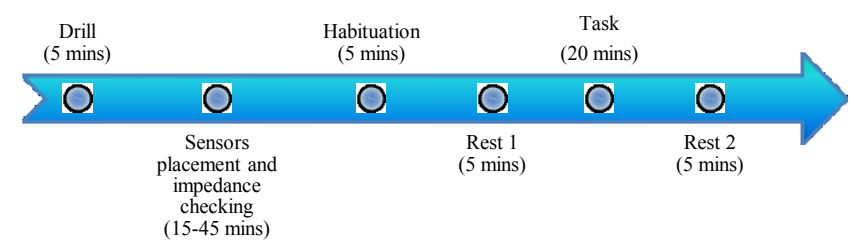

Figure 1. Experiment process

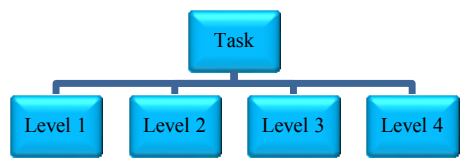

Figure 2. Task levels

Ten healthy male subjects (age: 19-25 years) were recruited in this study. They were selected based on criteria: previous medical record, i.e., only those were chosen who had no head injury and not using any medication that might increase cardiac activation. Subjects were asked to perform fasting for at least two hours before starting the experiment. Each subject signed an informed consent agreeing to participate, and was given an honorarium of RM 40 for his/her contribution.

\section{B. Data Acquisition and Analysis}

EEG and ECG data were simultaneously measured during the experiment. The data were acquired using Electrical Geodesic Inc. (EGI) Net Amps 300 amplifier and Net Station 4.4.5 acquisition software. EEG data was recorded using the 128 channels net with reference at $\mathrm{Cz}$ location at the sampling rate 500 samples/second. Reference of offline signals was changed to average mastoid. Impedance of all the electrodes was kept below $50 \mathrm{k} \Omega$. Two $\mathrm{Ag} / \mathrm{AgCl}$ surface electrodes were patched onto the bottom of the neck to measure ECG at 500 samples/second using the same system. ECG signals were later down sampled to 200 samples/second before HRV analysis.

EEG data was manually cleaned to remove artifacts. Sixty seconds of artifact free data was selected to keep the test retest reliability of data $95 \%$ [14]. HR analysis was performed on the five minute blocks of Rest 1 and Rest 2 and task levels (Level 1, 2, 3 and 4), respectively. The details of calculating the ratio $\mathrm{Fz}(\theta) / \mathrm{Pz}(\alpha)$ and $\mathrm{HR}$ can be found in [12].

Subject's performance was used to measure task difficulty in every level of experiment and was calculated by the formula shown in (1). Its value varied from 0 (min.) to 1 (max.). Out of three possible responses for every trial, the possibility of 'no response' increased during stress because of limited time for solving a trail. 'No response' reflected 
subject's being able to solve a trial but could not do because of time constrain. However, 'no response' had a rare occurrence during control session that was the reason it had been omitted from considering into the formula.

$$
\text { Task difficulty }=1-\frac{\text { Correct responses }}{\text { Total trials }}
$$

Paired t-test was applied on the results of the ratio $\mathrm{Fz}(\theta) / \mathrm{Pz}(\alpha), \mathrm{HR}$ and performance in order to measure significance. Paired t-test was applied between the ratio $\mathrm{Fz}(\theta) / \mathrm{Pz}(\alpha)$ and $\mathrm{HR}$ in every level in stress and control conditions vs. their respective rest 1 conditions. Same levels in stress and control were also tested for the ratio $\mathrm{Fz}(\theta) / \mathrm{Pz}(\alpha), \mathrm{HR}$ and performance.

\section{RESULTS}

\section{A. Performance Results}

Fig. 3 shows task difficulty in every task level of stress and control sessions. It was seen that under stress condition the difficulty of solving tasks was continuously increasing with every level $(0.42,0.5,0.59$ and 0.76$)$. Under control condition the difficulty in level 2 and 3 was slightly varied, but in other levels it increased continuously $(0.25,0.35,0.33$ and 0.53 ). It is prominent from the figure that difficulty in stress session is considerably higher than control session for all the levels. The subjects were less able to solve trials correctly in stress as compared to control condition. This difference of task difficulty under stress and control was significant $(p<0.003$ in all levels) which showed the poor performance and extreme trouble faced in stress condition.

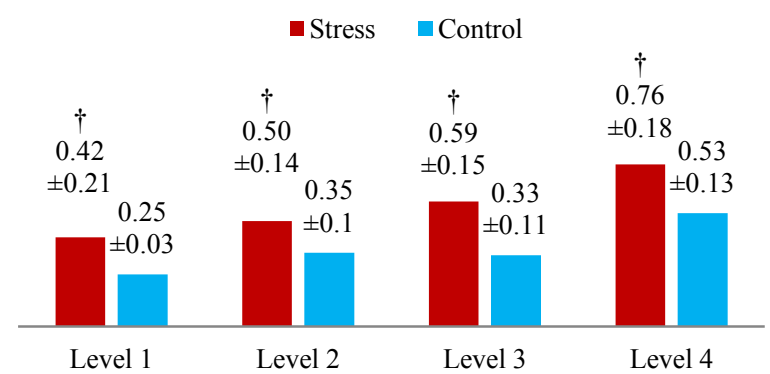

Figure 3. Task difficulty in level 1, 2, 3 and 4 in stress and control, values are arranged as mean \pm std, $\dagger$ indicates significance between stress and control conditions.

\section{B. EEG Results}

Fig. 4 presents the results of the ratio $\operatorname{Fz}(\theta) / \operatorname{Pz}(\alpha)$ for all the subjects during rest 1 , every task level (Level 1, Level 2, Level 3 and Level 4) and rest 2. The ratios showed significant difference of values in tasks as compared to the rest 1 baseline $(p<0.013)$, within stress and control sessions. The ratio also indicated significant difference between stress and control sessions for levels 1,2 and $3(\mathrm{p}<$ $0.042)$. The ratio in rest 1 was very precise for both the sessions (1.26 and 1.3 in stress and control respectively), which implied that the baseline for both conditions was almost same. In stress session, first three levels of stress continuously increased $(2.04,2.12$ and 2.31 in level 1, 2 and
3). However, in level 4, the ratio slightly decreased (2.25). The reduction may indicate that subjects reduced attempts of solving questions because of restricted time and extreme difficulty in level 4 of stress, which reduced the motor planning. Therefore, theta in Fz was reduced in power and it was reflected in the ratio in level 4 of stress. In control session, the ratio showed a similar pattern as shown by task difficulty. In the first two levels, the ratio increased (1.69 and 1.92 in level 1 and 2), then decreased for level 3 (1.84) and then again increased for level 4 (2.05). The reduction of the ratio in level 3 was unexplainable, but considerable increase of the ratio in level 4 had strengthen the possibility that reduced value of the ratio in level 4 of stress was because of limited time and extreme difficulty. When sufficient time was provided in level 4 of control, the subjects kept themselves involved in attempting the questions. The ratio in rest 2 in both conditions, revert back towards baseline level (1.36 and 1.31 in stress and control respectively), which showed that the subject recovered after the experiment.

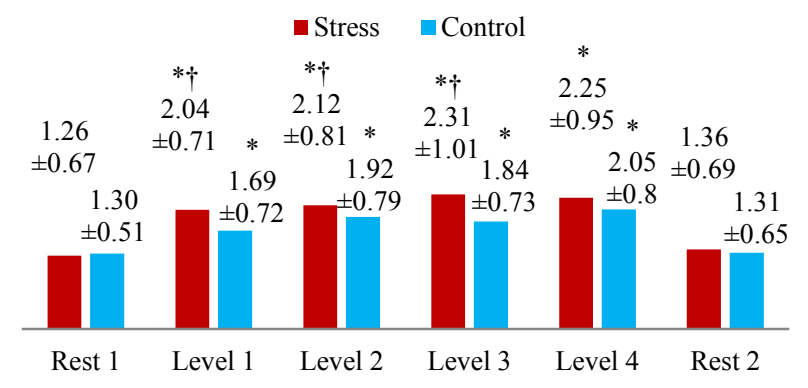

Figure 4. $\operatorname{Fz}(\theta) / \operatorname{Pz}(\alpha)$ values in level 1, 2, 3 and 4 in stress and control, values are arranged as mean $\pm \mathrm{std}, *$ indicates significance within stress and control conditions w.r.t. rest 1 and $\dagger$ indicates significance between stress and control conditions

\section{HR Results}

Fig. 5 illustrates the results of HR in all the levels of stress and control sessions. It was seen that HR had significantly increased during all task levels within stress and control sessions with respect to their respective rest $1(p<0.05$ in all levels of stress and control). However, HR did not show any significance between stress and control conditions.

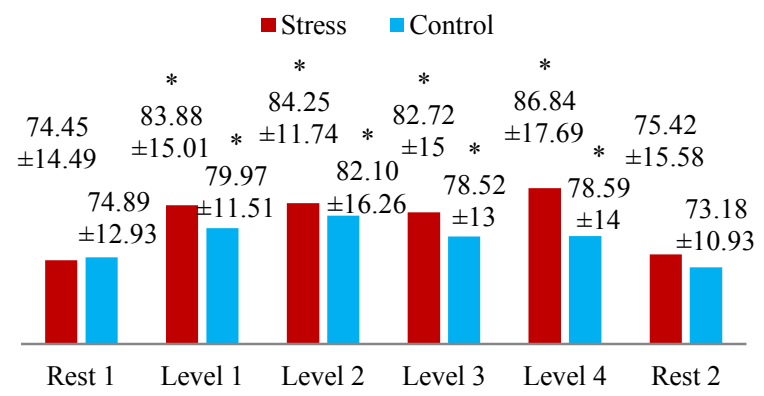

Figure 5. Heart rate in level 1, 2, 3 and 4 in stress and control, values are arranged as mean $\pm \mathrm{std}$, * indicates significance within stress and control conditions w.r.t. rest 1 .

Baseline (Rest 1) of both sessions was very close (74.45 and 74.89 beats per min. (bpm) in stress and control). For 
stress session, HR increased for first two levels (83.88 and $84.25 \mathrm{bpm})$. However, HR reduced down in level 3 (82.72 bpm) and increased again in level 4 (86.84 bpm) which was marked as the highest HR among all the sessions. HR in control session showed a similar pattern to the HR in stress, i.e., in first two levels of control HR increased (79.97 and $82.1 \mathrm{bpm}$, respectively) which reduced in level 3 (78.52 bpm). However, in level 4 of control, unlike the level 4 of stress, HR was almost same as level 3 (78.59 bpm). After the tasks were finished, HR approached back to baseline in both sessions (75.42 and $73.18 \mathrm{bpm}$ in stress and control).

\section{Discussion}

The results presented significant changes in HR $(p<0.05)$ in association with the variation in EEG scalp potentials $(\mathrm{p}<0.013)$ in every task level within stress and control sessions with respect to their respective baselines. These findings showed a considerable sympathetic innervation of heart along with higher activation of scalp potential during both sessions. However, the disparities during stress were even more than control session. As discussed earlier sympathetic activity had excitatory behavior to the heart. In this case, surge in HR had been consistently higher in every level during stress session than control session. Cardiac excitation was accompanied with cortical activation. The ratio of $\mathrm{Fz}(\theta) / \mathrm{Pz}(\alpha)$ was significantly higher during stress tasks than control tasks, particularly in levels 1,2 and 3 $(\mathrm{p}<0.042)$. This activation indicated a remarkable workload during stress session compared to control session. The achieved results have provided positive direction since it is reported in [11] that short range coherence in theta increases with difficulty in anterior region. As the baselines (Rest 1) and recovery (Rest 2) of both the sessions had very close values of HR and workload index, any higher variation during stress session than control session can be declared because of induction of stress in former session.

In addition, task difficulty derived from subjects' performance during the experiment was significantly higher in stress session than control session $(p<0.003)$. This considerable difficulty in performing task in stressful environment reflects the miserable performance of subjects under stress.

\section{CONCLUSION}

We observe a prominent increase in the scalp potential during stress task in conjunction with sympathetic activation. Higher ratio of $\mathrm{Fz} / \mathrm{Pz}$ in stress tasks physiologically represents considerable workload. At the same time, higher HR values in stress show more sympathetic activation than baseline and control. These findings are in association with the task difficulty during stress. The results support the idea to observe cerebral activation and autonomic innervations to measure mental stress. Finally, increased task difficulty consolidates the idea that growing demands deteriorate the individual's performance.
In future, more samples will be added in this study quantitative analysis will be performed on EEG data.

\section{ACKNOWLEDGMENT}

This work is supported by the Fundamental Research Grant Scheme (Ref: FRGS/1/2012/TK02/UTP/03/03), Ministry of High Education (MOHE), Malaysia and URIF (Ref: URIF 12/2012), Universiti Teknologi PETRONAS, Malaysia

\section{REFERENCES}

K. Dedovic, R. Renwick, N. K. Mahani, V. Engert, S. J. Lupien, and J. C. Pruessner, "The Montreal Imaging Stress Task: Using functional imaging to investigate the effects of perceiving and processing psychosocial stress in the human brain," Journal of Psychiatry and Neuroscience, vol. 30, pp. 319-325, 2005.

[2] Walter J. Hendelman, Peter Humphreys, and Christopher R. Skinner, The Integrated Nervous System: A Systematic Diagnostic Approach Boca Raton: CRC Press, 2010.

[3] J. E. Walker, G. P. Kozlowski, and R. Lawson, "A modular activation/coherence approach to evaluating clinical/QEEG correlations and for guiding neurofeedback training: Modular insufficiencies, modular excesses, disconnections, and hyperconnections," Journal of Neurotherapy, vol. 11, pp. 25-44, 2007.

[4] A. Holm, K. Lukander, J. Korpela, M. Sallinen, and K. M. I. Müller, "Estimating brain load from the EEG," TheScientificWorldJournal, vol. 9, pp. 639-651, 2009.

[5] J. A. Healey and R. W. Picard, "Detecting stress during realworld driving tasks using physiological sensors," IEEE Transactions on Intelligent Transportation Systems, vol. 6, pp. 156-166, 2005.

[6] C. Setz, B. Arnrich, J. Schumm, R. La Marca, G. Tröster, and U. Ehlert, "Discriminating stress from cognitive load using a wearable eda device," IEEE Transactions on Information Technology in Biomedicine, vol. 14, pp. 410-417, 2010.

[7] J. R. Carter and C. A. Ray, "Sympathetic neural responses to mental stress: Responders, nonresponders and sex differences," American Journal of Physiology - Heart and Circulatory Physiology, vol. 296, pp. H847-H853, 2009.

[8] X. L. Yu, J. B. Zhang, D. D. Xie, J. Wang, and C. Zhang, "Relationship between scalp potential and autonomic nervous activity during a mental arithmetic task," Autonomic Neuroscience-Basic \& Clinical, vol. 146, pp. 81-86, Mar 2009.

[9] C. L. Baldwin and B. N. Penaranda, "Adaptive training using an artificial neural network and EEG metrics for within- and crosstask workload classification," NeuroImage, vol. 59, pp. 48-56, 2012.

[10] K. Ryu and R. Myung, "Evaluation of mental workload with a combined measure based on physiological indices during a dual task of tracking and mental arithmetic," International Journal of Industrial Ergonomics, vol. 35, pp. 991-1009, 2005.

[11] P. Sauseng, W. Klimesch, M. Schabus, and M. Doppelmayr, "Fronto-parietal EEG coherence in theta and upper alpha reflect central executive functions of working memory," International Journal of Psychophysiology, vol. 57, pp. 97-103, 2005.

[12] A. R. Subhani, L. Xia, and A. Saeed Malik, "Association of autonomic nervous system and EEG scalp potential during playing 2D grand turismo 5," in 34th Annual International Conference of the IEEE Engineering in Medicine and Biology Society, San Diego, CA, pp. 3420-3423, 2012.

[13] A. R. Subhani, L. Xia, and A. S. Malik, "Association of Electroencephalogram Scalp Potential with Autonomic Nervous System," Advanced Science Letters, vol. 19, pp. 1340-1344, 2012.

[14] T. H. Budzynski, H. K. Budzynski, J. R. Evans, and A. Abarbanel, Introduction to Quantitative EEG and Neurofeedback: Advanced Theory and Applications, Second ed., 2009. 